EUROPEAN ORGANISATION FOR NUCLEAR RESEARCH

CERN-PPE/96-182

13 December 1996

\title{
Search for Charged Scalar Leptons Using the OPAL Detector at $\sqrt{s}=161 \mathrm{GeV}$
}

\author{
The OPAL Collaboration
}

\begin{abstract}
A search for pair-produced scalar electron, muon and tau leptons has been performed using a data sample corresponding to an integrated luminosity of $10.1 \mathrm{pb}^{-1}$ at a centreof-mass energy of $\sqrt{s}=161 \mathrm{GeV}$ collected with the OPAL detector at LEP. Such events would yield an acoplanar pair of leptons with significant missing energy in the final state. No excess of acoplanar lepton pair events has been observed in the data. Limits are presented on the production cross-sections of charged scalar leptons. Exclusion regions are also presented in the framework of the Minimal Supersymmetric Standard Model. A $95 \%$ C.L. lower limit on the right-handed selectron mass of $71.5 \mathrm{GeV}$ is obtained for $\tan \beta \geq 1.5,|\mu| \geq 200 \mathrm{GeV}$ and $m_{\tilde{\chi}_{1}^{0}}=12.0 \mathrm{GeV}$. A $95 \%$ C.L. lower limit on the righthanded smuon mass of $51.0 \mathrm{GeV}$ is obtained for $m_{\tilde{\chi}_{1}^{0}}=12.0 \mathrm{GeV}$, independent of $\tan \beta$ and $\mu$.
\end{abstract}




\section{The OPAL Collaboration}

K. Ackerstaff ${ }^{8}$, G. Alexander ${ }^{23}$, J. Allison ${ }^{16}$, N. Altekamp ${ }^{5}$, K. Ametewee ${ }^{25}$, K.J. Anderson ${ }^{9}$,

S. Anderson ${ }^{12}$, S. Arcelli ${ }^{2}$, S. Asai ${ }^{24}$, D. Axen ${ }^{29}$, G. Azuelos ${ }^{18, a}$, A.H. Ball ${ }^{17}$, E. Barberio ${ }^{8}$,

R.J. Barlow ${ }^{16}$, R. Bartoldus ${ }^{3}$, J.R. Batley ${ }^{5}$, J. Bechtluft ${ }^{14}$, C. Beeston ${ }^{16}$, T. Behnke ${ }^{8}$, A.N. Bell ${ }^{1}$,

K.W. Bell ${ }^{20}$, G. Bella ${ }^{23}$, S. Bentvelsen ${ }^{8}$, P. Berlich ${ }^{10}$, S. Bethke ${ }^{14}$, O. Biebel ${ }^{14}$, A. Biguzzi ${ }^{2}$,

V.Blobel $^{27}$, I.J. Bloodworth ${ }^{1}$, J.E. Bloomer ${ }^{1}$, M. Bobinski ${ }^{10}$, P. Bock ${ }^{11}$, H.M. Bosch ${ }^{11}$,

M. Boutemeur ${ }^{34}$, B.T. Bouwens ${ }^{12}$, S. Braibant ${ }^{12}$, R.M. Brown ${ }^{20}$, H.J. Burckhart ${ }^{8}$, C. Burgard $^{8}$,

R. Bürgin ${ }^{10}$, P. Capiluppi ${ }^{2}$, R.K. Carnegie ${ }^{6}$, A.A. Carter ${ }^{13}$, J.R. Carter ${ }^{5}$, C.Y. Chang ${ }^{17}$,

D.G. Charlton ${ }^{1, b}$, D. Chrisman ${ }^{4}$, P.E.L. Clarke ${ }^{15}$, I. Cohen ${ }^{23}$, J.E. Conboy ${ }^{15}$, O.C. Cooke ${ }^{16}$,

M. Cuffiani ${ }^{2}$, S. Dado ${ }^{22}$, C. Dallapiccola ${ }^{17}$, G.M. Dallavalle ${ }^{2}$, S. De Jong ${ }^{12}$, L.A. del Pozo ${ }^{8}$,

K. Desch ${ }^{3}$, M.S. Dixit ${ }^{7}$, E. do Couto e Silva ${ }^{12}$, M. Doucet ${ }^{18}$, E. Duchovni ${ }^{26}$, G. Duckeck ${ }^{34}$,

I.P. Duerdoth ${ }^{16}$, J.E.G. Edwards ${ }^{16}$, P.G. Estabrooks $^{6}$, H.G. Evans ${ }^{9}$, M. Evans ${ }^{13}$, F. Fabbri ${ }^{2}$,

P. Fath ${ }^{11}$, F. Fiedler ${ }^{27}$, M. Fierro ${ }^{2}$, H.M. Fischer ${ }^{3}$, R. Folman ${ }^{26}$, D.G. Fong ${ }^{17}$, M. Foucher ${ }^{17}$,

A. Fürtjes ${ }^{8}$, P. Gagnon ${ }^{7}$, J.W. Gary ${ }^{4}$, J. Gascon ${ }^{18}$, S.M. Gascon-Shotkin ${ }^{17}$, N.I. Geddes ${ }^{20}$,

C. Geich-Gimbel ${ }^{3}$, T. Geralis ${ }^{20}$, G. Giacomelli ${ }^{2}$, P. Giacomelli ${ }^{4}$, R. Giacomelli ${ }^{2}$, V. Gibson ${ }^{5}$,

W.R. Gibson ${ }^{13}$, D.M. Gingrich ${ }^{30, a}$, D. Glenzinski ${ }^{9}$, J. Goldberg ${ }^{22}$, M.J. Goodrick ${ }^{5}$, W. Gorn ${ }^{4}$,

C. Grandi ${ }^{2}$, E. Gross ${ }^{26}$,J. Grunhaus ${ }^{23}$ M. Gruwé ${ }^{8}$, C. Hajdu ${ }^{32}$, G.G. Hanson ${ }^{12}$, M. Hansroul ${ }^{8}$,

M. Hapke ${ }^{13}$, C.K.Hargrove ${ }^{7}$, P.A.Hart ${ }^{9}$, C.Hartmann ${ }^{3}$, M. Hauschild ${ }^{8}$, C.M. Hawkes ${ }^{5}$,

R. Hawkings ${ }^{8}$, R.J. Hemingway ${ }^{6}$, M. Herndon ${ }^{17}$, G. Herten ${ }^{10}$, R.D. Heuer ${ }^{8}$, M.D. Hildreth ${ }^{8}$, J.C. Hill ${ }^{5}$, S.J. Hillier ${ }^{1}$, T. Hilse ${ }^{10}$, P.R.Hobson ${ }^{25}$, R.J.Homer ${ }^{1}$, A.K. Honma ${ }^{28, a}$, D. Horváth ${ }^{32, c}$,

R. Howard ${ }^{29}$, R.E. Hughes-Jones ${ }^{16}$, D.E. Hutchcroft ${ }^{5}$, P. Igo-Kemenes ${ }^{11}$, D.C. Imrie ${ }^{25}$,

M.R. Ingram ${ }^{16}$, K. Ishii ${ }^{4}$, A. Jawahery ${ }^{17}$, P.W. Jeffreys ${ }^{20}$, H. Jeremie ${ }^{18}$, M. Jimack ${ }^{1}$, A. Joly ${ }^{18}$,

C.R. Jones ${ }^{5}$, G. Jones ${ }^{16}$, M. Jones ${ }^{6}$, R.W.L. Jones ${ }^{8}$, U. Jost ${ }^{11}$, P. Jovanovic ${ }^{1}$, T.R. Junk ${ }^{8}$,

D. Karlen ${ }^{6}$, K. Kawagoe ${ }^{24}$, T. Kawamoto ${ }^{24}$, R.K. Keeler ${ }^{28}$, R.G. Kellogg ${ }^{17}$, B.W. Kennedy ${ }^{20}$,

B.J. King ${ }^{8}$, J. Kirk ${ }^{29}$, S. Kluth ${ }^{8}$, T. Kobayashi ${ }^{24}$, M. Kobel ${ }^{10}$, D.S. Koetke ${ }^{6}$, T.P. Kokott ${ }^{3}$,

M. Kolrep ${ }^{10}$, S. Komamiya ${ }^{24}$, T. Kress ${ }^{11}$, P. Krieger ${ }^{6}$, J. von Krogh ${ }^{11}$, P. Kyberd ${ }^{13}$,

G.D. Lafferty ${ }^{16}$, R. Lahmann ${ }^{17}$, W.P. Lai ${ }^{19}$, D. Lanske ${ }^{14}$, J. Lauber ${ }^{15}$, S.R. Lautenschlager ${ }^{31}$,

J.G. Layter ${ }^{4}$, D. Lazic ${ }^{22}$, A.M. Lee ${ }^{31}$, E. Lefebvre ${ }^{18}$, D. Lellouch ${ }^{26}$, J. Letts ${ }^{2}$, L. Levinson ${ }^{26}$,

C. Lewis ${ }^{15}$, S.L. Lloyd ${ }^{13}$, F.K. Loebinger ${ }^{16}$, G.D. Long ${ }^{17}$, M.J. Losty ${ }^{7}$, J. Ludwig ${ }^{10}$,

M. Mannelli ${ }^{8}$, S. Marcellini ${ }^{2}$, C. Markus ${ }^{3}$, A.J. Martin ${ }^{13}$, J.P. Martin ${ }^{18}$, G. Martinez ${ }^{17}$,

T. Mashimo ${ }^{24}$, W. Matthews ${ }^{25}$, P. Mättig ${ }^{3}$, W.J. McDonald ${ }^{30}$, J. McKenna ${ }^{29}$, E.A. Mckigney ${ }^{15}$,

T.J. McMahon ${ }^{1}$, A.I. McNab ${ }^{13}$, R.A. McPherson ${ }^{8}$, F. Meijers ${ }^{8}$, S. Menke ${ }^{3}$, F.S. Merritt ${ }^{9}$,

H. Mes ${ }^{7}$, J. Meyer ${ }^{27}$, A. Michelini ${ }^{2}$, G. Mikenberg ${ }^{26}$, D.J. Miller ${ }^{15}$, R. Mir ${ }^{26}$, W. Mohr ${ }^{10}$,

A. Montanari ${ }^{2}$, T. Mori ${ }^{24}$, M. Morii ${ }^{24}$, U.Müller ${ }^{3}$, K. Nagai $^{26}$, I. Nakamura ${ }^{24}$, H.A. Neal ${ }^{8}$,

B. Nellen ${ }^{3}$, B. Nijjhar ${ }^{16}$, R. Nisius ${ }^{8}$, S.W. O'Neale ${ }^{1}$, F.G.Oakham ${ }^{7}$, F. Odorici ${ }^{2}$, H.O. Ogren ${ }^{12}$,

N.J. Oldershaw ${ }^{16}$, T. Omori ${ }^{24}$, M.J. Oreglia ${ }^{9}$, S. Orito ${ }^{24}$, J. Pálinkás ${ }^{33, d}$, G. Pásztor ${ }^{32}$,

J.R.Pater ${ }^{16}$, G.N.Patrick ${ }^{20}$, J. Patt ${ }^{10}$, M.J. Pearce ${ }^{1}$, S. Petzold ${ }^{27}$, P. Pfeifenschneider ${ }^{14}$,

J.E. Pilcher ${ }^{9}$, J. Pinfold ${ }^{30}$, D.E. Plane ${ }^{8}$, P. Poffenberger ${ }^{28}$, B. Poli ${ }^{2}$, A. Posthaus ${ }^{3}$,

H. Przysiezniak ${ }^{30}$, D.L. Rees ${ }^{1}$, D. Rigby ${ }^{1}$, S. Robertson ${ }^{28}$, S.A. Robins ${ }^{13}$, N. Rodning ${ }^{30}$, J.M. Roney ${ }^{28}$, A. Rooke ${ }^{15}$, E. $\operatorname{Ros}^{8}$, A.M. Rossi ${ }^{2}$, M. Rosvick ${ }^{28}$, P. Routenburg ${ }^{30}$, Y. Rozen ${ }^{22}$,

K. Runge ${ }^{10}$, O. Runolfsson ${ }^{8}$, U. Ruppel ${ }^{14}$, D.R.Rust ${ }^{12}$, R. Rylko ${ }^{25}$, K.Sachs $^{10}$,

E.K.G.Sarkisyan ${ }^{23}$, M.Sasaki ${ }^{24}$, C.Sbarra ${ }^{2}$, A.D.Schaile ${ }^{34}$, O.Schaile ${ }^{34}$, F. Scharf ${ }^{3}$,

P. Scharff-Hansen ${ }^{8}$, P. Schenk ${ }^{27}$, B. Schmitt ${ }^{8}$, S. Schmitt ${ }^{11}$, M. Schröder ${ }^{8}$,

H.C.Schultz-Coulon ${ }^{10}$, M.Schulz ${ }^{8}$, M.Schumacher ${ }^{3}$, P.Schütz ${ }^{3}$, W.G.Scott ${ }^{20}$, T.G.Shears ${ }^{16}$,

B.C.Shen ${ }^{4}$, C.H. Shepherd-Themistocleous ${ }^{8}$, P. Sherwood ${ }^{15}$, G.P.Siroli ${ }^{2}$, A.Sittler ${ }^{27}$, 
A.Skillman ${ }^{15}$, A.Skuja ${ }^{17}$, A.M.Smith ${ }^{8}$, T.J.Smith ${ }^{28}$, G.A.Snow ${ }^{17}$, R. Sobie ${ }^{28}$, S. Söldner-Rembold ${ }^{10}$, R.W.Springer ${ }^{30}$, M.Sproston ${ }^{20}$, A.Stahl ${ }^{3}$, M. Steiert ${ }^{11}$, K.Stephens ${ }^{16}$, J.Steuerer ${ }^{27}$, B.Stockhausen ${ }^{3}$, D.Strom ${ }^{19}$, F.Strumia ${ }^{8}$, P.Szymanski ${ }^{20}$, R. Tafirout ${ }^{18}$, S.D. Talbot ${ }^{1}$, S. Tanaka ${ }^{24}$, P. Taras ${ }^{18}$, S. Tarem ${ }^{22}$, M. Thiergen ${ }^{10}$, M.A. Thomson ${ }^{8}$, E. von

Törne $^{3}$, S. Towers ${ }^{6}$, I. Trigger ${ }^{18}$, T. Tsukamoto ${ }^{24}$, E. Tsur ${ }^{23}$, A.S. Turcot ${ }^{9}$,

M.F. Turner-Watson ${ }^{8}$, P. Utzat ${ }^{11}$, R. Van Kooten ${ }^{12}$, M. Verzocchi ${ }^{10}$, P. Vikas ${ }^{18}$, M. Vincter ${ }^{28}$,

E.H. Vokurka ${ }^{16}$, F. Wäckerle ${ }^{10}$, A. Wagner ${ }^{27}$, C.P. Ward ${ }^{5}$, D.R. Ward ${ }^{5}$, J.J. Ward ${ }^{15}$,

P.M. Watkins ${ }^{1}$, A.T. Watson ${ }^{1}$, N.K. Watson ${ }^{7}$, P.S. Wells ${ }^{8}$, N. Wermes ${ }^{3}$, J.S. White ${ }^{28}$,

B. Wilkens ${ }^{10}$, G.W.Wilson ${ }^{27}$, J.A.Wilson ${ }^{1}$, G. Wolf ${ }^{26}$, S. Wotton ${ }^{5}$, T.R. Wyatt ${ }^{16}$,

S. Yamashita ${ }^{24}$, G. Yekutieli ${ }^{26}$, V. Zacek ${ }^{18}$

${ }^{1}$ School of Physics and Space Research, University of Birmingham, Birmingham B15 2TT, UK

${ }^{2}$ Dipartimento di Fisica dell' Università di Bologna and INFN, I-40126 Bologna, Italy

${ }^{3}$ Physikalisches Institut, Universität Bonn, D-53115 Bonn, Germany

${ }^{4}$ Department of Physics, University of California, Riverside CA 92521, USA

${ }^{5}$ Cavendish Laboratory, Cambridge CB3 0HE, UK

${ }^{6}$ Ottawa-Carleton Institute for Physics, Department of Physics, Carleton University, Ottawa, Ontario K1S 5B6, Canada

${ }^{7}$ Centre for Research in Particle Physics, Carleton University, Ottawa, Ontario K1S 5B6, Canada

${ }^{8}$ CERN, European Organisation for Particle Physics, CH-1211 Geneva 23, Switzerland

${ }^{9}$ Enrico Fermi Institute and Department of Physics, University of Chicago, Chicago IL 60637, USA

${ }^{10}$ Fakultät für Physik, Albert Ludwigs Universität, D-79104 Freiburg, Germany

${ }^{11}$ Physikalisches Institut, Universität Heidelberg, D-69120 Heidelberg, Germany

${ }^{12}$ Indiana University, Department of Physics, Swain Hall West 117, Bloomington IN 47405, USA

${ }^{13}$ Queen Mary and Westfield College, University of London, London E1 4NS, UK

${ }^{14}$ Technische Hochschule Aachen, III Physikalisches Institut, Sommerfeldstrasse 26-28, D-52056 Aachen, Germany

${ }^{15}$ University College London, London WC1E 6BT, UK

${ }^{16}$ Department of Physics, Schuster Laboratory, The University, Manchester M13 9PL, UK

${ }^{17}$ Department of Physics, University of Maryland, College Park, MD 20742, USA

${ }^{18}$ Laboratoire de Physique Nucléaire, Université de Montréal, Montréal, Quebec H3C 3J7, Canada

${ }^{19}$ University of Oregon, Department of Physics, Eugene OR 97403, USA

${ }^{20}$ Rutherford Appleton Laboratory, Chilton, Didcot, Oxfordshire OX11 0QX, UK

${ }^{22}$ Department of Physics, Technion-Israel Institute of Technology, Haifa 32000, Israel

${ }^{23}$ Department of Physics and Astronomy, Tel Aviv University, Tel Aviv 69978, Israel

${ }^{24}$ International Centre for Elementary Particle Physics and Department of Physics, University of Tokyo, Tokyo 113, and Kobe University, Kobe 657, Japan

${ }^{25}$ Brunel University, Uxbridge, Middlesex UB8 3PH, UK

${ }^{26}$ Particle Physics Department, Weizmann Institute of Science, Rehovot 76100, Israel

${ }^{27}$ Universität Hamburg/DESY, II Institut für Experimental Physik, Notkestrasse 85, D-22607

Hamburg, Germany

${ }^{28}$ University of Victoria, Department of Physics, P O Box 3055, Victoria BC V8W 3P6, Canada 
${ }^{29}$ University of British Columbia, Department of Physics, Vancouver BC V6T 1Z1, Canada ${ }^{30}$ University of Alberta, Department of Physics, Edmonton AB T6G 2J1, Canada

${ }^{31}$ Duke University, Dept of Physics, Durham, NC 27708-0305, USA

${ }^{32}$ Research Institute for Particle and Nuclear Physics, H-1525 Budapest, P O Box 49, Hungary ${ }^{33}$ Institute of Nuclear Research, H-4001 Debrecen, P O Box 51, Hungary

${ }^{34}$ Ludwigs-Maximilians-Universität München, Sektion Physik, Am Coulombwall 1, D-85748 Garching, Germany

${ }^{a}$ and at TRIUMF, Vancouver, Canada V6T $2 \mathrm{~A} 3$

${ }^{b}$ and Royal Society University Research Fellow

${ }^{c}$ and Institute of Nuclear Research, Debrecen, Hungary

${ }^{d}$ and Department of Experimental Physics, Lajos Kossuth University, Debrecen, Hungary 


\section{Introduction}

In Supersymmetry (SUSY) [1] models each elementary particle is accompanied by a supersymmetric partner whose spin differs by half a unit. Each lepton has two scalar partners, the rightand left-handed scalar leptons (sleptons) denoted $\tilde{\ell}_{\mathrm{R}}$ and $\tilde{\ell}_{\mathrm{L}}$, according to the helicity states of their non-SUSY partners. In electron-positron collisions, sleptons could be pair-produced through $s$-channel processes involving a $\mathrm{Z}^{0}$ or a $\gamma$. Scalar electrons (selectrons, $\tilde{e}$ ) could also be produced through $t$-channel neutralino exchange enhancing the selectron production crosssection compared to those for the scalar muon (smuon, $\tilde{\mu}$ ) and scalar tau (stau, $\tilde{\tau}$ ). In the following we will make the assumption that a new multiplicative quantum number, R-parity [2], which discriminates between ordinary and supersymmetric particles, is conserved and that the lightest neutralino, $\tilde{\chi}_{1}^{0}$, is the lightest supersymmetric particle (LSP). These assumptions imply that supersymmetric particles are always pair-produced and always decay promptly through cascade decays to ordinary particles and $\tilde{\chi}_{1}^{0}$. The $\tilde{\chi}_{1}^{0}$ is stable and escapes detection due to its weakly interacting nature.

In this letter, we report on a direct search for events with an acoplanar pair of leptons with significant missing energy in the final state. This topology is the typical experimental signature for charged scalar lepton pair production:

$$
\begin{gathered}
\mathrm{e}^{+} \mathrm{e}^{-} \rightarrow \tilde{\ell}^{+} \tilde{\ell}^{-}, \\
\tilde{\ell}^{ \pm} \rightarrow \ell^{ \pm} \tilde{\chi}_{1}^{0},
\end{gathered}
$$

where $\ell^{ \pm}$is the corresponding charged lepton. For the purpose of our charged slepton searches, the more complicated topologies which would arise from cascade decays if the next-to-lightest neutralino, $\tilde{\chi}_{2}^{0}$, and the lightest charginos, $\tilde{\chi}_{1}^{ \pm}$, are lighter than the sleptons are not investigated as search topologies since the $\tilde{\ell}^{ \pm} \rightarrow \ell^{ \pm}+\tilde{\chi}_{1}^{0}$ decay mode is expected to be dominant in most of the cases.

In the following, the Minimal Supersymmetric extension of the Standard Model (MSSM) [3] is used as a reference model, although the results reported here are also valid in a broader context. In the framework of the MSSM, under the assumption of a common scalar mass $m_{0}$ at the Grand Unification (GUT) scale, all sparticle masses (but not the Higgs boson masses) and couplings are completely determined by $m_{0}$ and a set of three parameters: $M_{2}$, the $\mathrm{SU}(2)$ gaugino mass parameter at electroweak scales ${ }^{1} ; \mu$, the mixing parameter of the two Higgs doublets; and $\tan \beta=v_{2} / v_{1}$, the ratio of the vacuum expectation values for the two Higgs doublets. In these constrained MSSM models, right-handed sleptons are always lighter than their left-handed counterparts, and the cross-section for production of right-handed sleptons is usually smaller than that for left-handed ones of the same mass. The MSSM mass limits, under the assumption of a common scalar mass $m_{0}$ at the GUT scale, were conservatively calculated using cross-sections corresponding to right-handed slepton production. We have also computed limits on scalar lepton pair-production in the MSSM framework without the assumption of a common $m_{0}$ at the GUT scale, but instead under the assumption of mass degeneracy between right- and left-handed scalar lepton states.

We have used an analysis similar to the one described in reference [4] searching for events containing a pair of isolated electrons, muons or taus in association with significant missing

\footnotetext{
${ }^{1}$ We assume that $M_{1}$, the $\mathrm{U}(1)$ gaugino mass at electroweak scales, is related to $M_{2}$ by the usual gauge unification condition: $M_{1}=\frac{5}{3} \tan ^{2} \theta_{W} M_{2}$.
} 
momentum using $10.1 \mathrm{pb}^{-1}$ of data collected by the OPAL detector at a centre-of-mass energy ${ }^{2}$ $\sqrt{s}=161 \mathrm{GeV}$, in the first LEP2 data-taking during the summer of 1996. At this energy the background due to $\ell^{+} \ell^{-} \nu \bar{\nu}$ final states from $\mathrm{W}^{+} \mathrm{W}^{-}$pair-production is expected to be sizeable.

The results obtained here improve on the existing limits presented by the LEP collaborations $[4],[5]$.

\section{The OPAL Detector}

A complete description of the OPAL detector can be found in Ref. [6] and only a brief overview is given here.

The central detector consists of a system of tracking chambers providing charged particle tracking over $96 \%$ of the full solid angle $\mathrm{e}^{3}$ inside a $0.435 \mathrm{~T}$ uniform magnetic field parallel to the beam axis. It consists of a two-layer silicon microstrip vertex detector, a high precision drift chamber, a large volume jet chamber and a set of $z$ chambers measuring the track coordinates along the beam direction. A lead-glass electromagnetic (EM) calorimeter located outside the magnet coil covers the full azimuthal range with excellent hermeticity in the polar angle range of $|\cos \theta|<0.82$ for the barrel region and $0.81<|\cos \theta|<0.984$ for the endcap region. The magnet return yoke is instrumented for hadron calorimetry (HCAL) and consists of barrel and endcap sections along with pole tip detectors that together cover the region $|\cos \theta|<0.99$. Four layers of muon chambers cover the outside of the hadron calorimeter. Electromagnetic calorimeters close to the beam axis complete the geometrical acceptance down to $24 \mathrm{mrad}$, except for the regions where a tungsten shield is present to protect the detectors from synchrotron radiation. These include the forward detectors (FD) which are lead-scintillator sandwich calorimeters and, at smaller angles, silicon tungsten calorimeters (SW) [7] located on both sides of the interaction point. The gap between the endcap EM calorimeter and the FD is instrumented with an additional lead-scintillator electromagnetic calorimeter, called the gamma-catcher.

\section{Monte Carlo Simulation}

The Monte Carlo program SUSYGEN [8] was used to generate samples of simulated righthanded $\tilde{\mathbf{e}}^{+} \tilde{\mathbf{e}}^{-}, \tilde{\mu}^{+} \tilde{\mu}^{-}$and $\tilde{\tau}^{+} \tilde{\tau}^{-}$events for various slepton masses. The most important parameters for the slepton detection efficiency are the mass of the slepton, $m_{\tilde{\ell}}$, and the mass difference between the slepton and the lightest neutralino, $\Delta m \equiv\left(m_{\tilde{\ell}}-m_{\tilde{\chi}_{1}^{0}}\right)$. Samples of $\tilde{\ell}^{+} \tilde{\ell}^{-}$events were generated for 40 combinations of $\left(m_{\tilde{\ell}}, m_{\tilde{\chi}_{1}^{0}}\right)$ at $\sqrt{s}=161 \mathrm{GeV}$. At each point, $2000 \tilde{\mathbf{e}}^{+} \tilde{\mathbf{e}}^{-}$, $1000 \tilde{\mu}^{+} \tilde{\mu}^{-}$and $1000 \tilde{\tau}^{+} \tilde{\tau}^{-}$events were generated.

There are several sources of background to the slepton signals. Two-photon processes are the most important background for the case of a small difference between $m_{\tilde{\ell}}$ and $m_{\tilde{\chi}_{1}^{0}}$, since the total measured transverse momentum relative to the beam direction for the signal becomes small and similar to that of two-photon processes. The Vermaseren [9] generator was used to

\footnotetext{
${ }^{2}$ Although our best estimate for the centre-of-mass energy is $161.3 \pm 0.2 \mathrm{GeV}$, the nominal energy was used in this analysis.

${ }^{3}$ The OPAL coordinate system is defined so that the $z$ axis is in the direction of the electron beam, the $x$ axis is horizontal and points towards the centre of the LEP ring, and $\theta$ and $\phi$ are the polar and azimuthal angles, defined relative to the $+z$ - and $+x$-axes, respectively. The radial coordinate is denoted as $r$.
} 
estimate the background contribution from all $\mathrm{e}^{+} \mathrm{e}^{-} \ell^{+} \ell^{-}$final states. Four-fermion processes constitute a serious potential background. We used the PYTHIA [10] and PHOJET [11] Monte Carlo generators to simulate hadronic events from two-photon processes where the virtual mass $Q^{2}$ of both photons is smaller than $1 \mathrm{GeV}^{2}$ and the invariant mass squared of the photon-photon system $\left(m_{\gamma \gamma}^{2}\right)$ is greater than $4 \mathrm{GeV}^{2}$. For events with higher $Q^{2}$, the PYTHIA generator was also used, with the condition $m_{\gamma \gamma}^{2}>3 \mathrm{GeV}^{2}$.

Four-fermion processes with two neutrinos and two electrons or muons in the final state also constitute a potentially serious background since their cross-sections are large at centre-of-mass energies above the $\mathrm{W}^{+} \mathrm{W}^{-}$threshold. We used the EXCALIBUR [15] generator to simulate events for all types of four-fermion processes, including $\mathrm{W}^{+} \mathrm{W}^{-}$pair production. The PYTHIA generator was used to simulate $\mathrm{e}^{+} \mathrm{e}^{-} \rightarrow(\gamma / \mathrm{Z})^{*} \mathrm{e}^{+} \mathrm{e}^{-}$events and was also used to simulate multihadronic events, although these events are not expected to contribute significantly to the background.

The KORALZ [12] event generator was used for the generation of $\mu^{+} \mu^{-}(\gamma)$ and $\tau^{+} \tau^{-}(\gamma)$ events and the BHWIDE [13] event generator was used for the generation of $\mathbf{e}^{+} \mathbf{e}^{-}(\gamma)$ events. $\tau^{+} \tau^{-}(\gamma)$ events are a potential source of background for the topology of two acollinear leptons because neutrinos emitted from $\tau$-decays carry away energy and momentum. The contributions from $\nu \bar{\nu} \gamma \gamma$ final states were evaluated using the NUNUGPV [14] event generator.

All generated events were processed through the full simulation of the OPAL detector [16], and the same event analysis chain was applied to simulated events as to the data.

\section{Description of the Analysis}

The analysis has been designed to maintain good efficiency while reducing the overall background, mainly due to $\ell^{+} \ell^{-} \nu \bar{\nu}$ final states from $\mathrm{W}^{+} \mathrm{W}^{-}$pair production, to a low level.

\subsection{Event Preselection}

To be considered in the analysis, tracks in the central detector and clusters in the electromagnetic calorimeter were required to satisfy the normal quality criteria employed in the analysis of Standard Model lepton pairs [17].

First, we applied multihadronic, cosmic and Bhabha scattering vetoes:

- Events were rejected if $N_{\text {trk }}+N_{\text {clus }}>18$, where $N_{\text {trk }}$ is the number of tracks in the central detector and $N_{\text {clus }}$ is the number of clusters in the electromagnetic calorimeter.

- Cosmic ray events were rejected using an algorithm described in the analysis of Standard Model muon pairs [17].

- Bhabha scattering events were identified and then vetoed as described in [18].

At the preselection level, it was also required that the ratio of the number of tracks satisfying the quality criteria to the total number of reconstructed tracks is greater than 0.2 in order to reduce backgrounds from beam-gas and beam-wall events. The visible energy, the visible mass 
and the total transverse momentum of the event were calculated using the method described in [19]. Tracks from converted photons were rejected using the algorithm employed in the analysis of Standard Model muon pairs [17]. Finally, the number of good charged tracks was required to be at least two and the minimum event missing transverse momentum $p_{t}^{\text {miss }}$ to be greater than $2 \mathrm{GeV}$.

\subsection{Lepton Identification}

Only tracks with $|\cos \theta|<0.95$ were considered for lepton identification.

Electrons A track was selected as an electron candidate if any of the following criteria were satisfied:

- The output probability of a neural net algorithm as described in [20] was greater than 0.8 .

- A standard electron selection algorithm as described in [21] for the barrel region or in $[22]$ for the endcap region was satisfied.

- $0.5<E / p<2.0$, where $p$ is the momentum of the electron candidate and $E$ is the energy of the electromagnetic calorimeter cluster associated with the track.

Muons A track was selected as a muon candidate according to the criteria employed in the analysis of Standard Model muon pairs [17]. That is, the track had associated activity in the muon chambers or hadron calorimeter strips or it had a high momentum but was associated with only a small energy deposit in the electromagnetic calorimeter.

Taus Tau candidates were selected by requiring that:

- There were at most three tracks within a cone of $35^{\circ}$ half-opening angle centred on a track.

- The invariant mass computed using all good tracks and EM clusters within the above cone was less than $3 \mathrm{GeV}$.

For muon and electron candidates the momentum was estimated from the charged track momentum measured in the central detector while for tau candidates the momentum was estimated from the vector sum of the measured momenta of the charged tracks within the tau cone.

\subsection{Event Selection}

To select slepton candidates, the following criteria were applied:

(1) To reduce the background from two-photon processes and multihadronic events, the total energy deposited in each silicon tungsten calorimeter had to be less than $5 \mathrm{GeV}$, less than $2 \mathrm{GeV}$ in each forward calorimeter, and less than $5 \mathrm{GeV}$ in each side of the gamma-catcher. 
(2) The background from two-photon processes and "radiative return" events $\left(\mathrm{e}^{+} \mathrm{e}^{-} \rightarrow \mathrm{Z} \gamma\right.$, where the $\gamma$ escapes down the beam pipe) was reduced by requiring that the polar angle of the missing momentum direction, $\theta_{\text {miss }}$, satisfied $\left|\cos \theta_{\text {miss }}\right|<0.9$. Figure $1(\mathrm{a})$ shows the $\left|\cos \theta_{\text {miss }}\right|$ distribution after cut (1) has been applied and requiring that the event contains two identified leptons.

(3) To further reduce the residual background from Standard Model lepton pair events, it was also required that $M_{\text {vis }} / \sqrt{s}<0.80$, where $M_{\text {vis }}$ is the event visible mass.

(4) Events had to contain exactly two well-identified and oppositely-charged leptons each with a transverse momentum with respect to the beam axis greater than $1 \mathrm{GeV}$. Figure $1(\mathrm{~b})$ shows the lepton transverse momentum distribution after cuts (1) to (3) have been applied and requiring that the event contains two identified leptons.

(5) The acoplanarity angle ${ }^{4}\left(\phi_{\text {acop }}\right)$ between the two leptons was required to be greater than $10^{\circ}$ in order to reject Standard Model leptonic events, and smaller than $175^{\circ}$ in order to reduce the background due to photon conversions. The acoplanarity angle distribution is shown in Figure $1(\mathrm{c})$ after cuts $(1)$ to (4). The acollinearity angle ${ }^{5}\left(\phi_{\text {acol }}\right)$ was also required to be greater than $10^{\circ}$ and smaller than $175^{\circ}$.

(6) To reduce the background due to radiative leptonic events it was required that there be no unassociated electromagnetic cluster with an energy larger than $25 \mathrm{GeV}$ in the event.

(7) In order to reduce occasional two-photon events with high transverse momentum, different cuts on the missing momentum and its direction were applied in the regions of small and large acoplanarity. For events with small acoplanarity $\left(\phi_{\text {acop }}<1.2 \mathrm{rad}\right)$, a cut on $a_{t}^{\text {miss }}$ was applied, where $a_{t}^{\text {miss }}$ is the component of the missing momentum vector perpendicular to the event thrust axis in the plane transverse to the beam axis. For events with large acoplanarity $\left(\phi_{\text {acop }}>1.2 \mathrm{rad}\right)$, a cut on $p_{t}^{\text {miss }}$ was applied. A further subdivision according to the visible energy of the event $\left(E_{\mathrm{vis}}\right)$ was applied in order to optimize the selection efficiencies for the entire possible range of $\Delta m$ varying from $2.5 \mathrm{GeV}$ to $80 \mathrm{GeV}$.

1. Cuts in the small acoplanarity region:

(a) If $E_{\text {vis }} / \sqrt{s}<0.06$, then $a_{t}^{\text {miss }} / \sqrt{s}>0.015$.

(b) If $E_{\text {vis }} / \sqrt{s}>0.06$, then $a_{t}^{\text {miss }} / \sqrt{s}>0.020$.

2. Cuts in the large acoplanarity region:

(a) If $E_{\text {vis }} / \sqrt{s}<0.06$, then $p_{t}^{\text {miss }} / \sqrt{s}>0.025$.

(b) If $E_{\text {vis }} / \sqrt{s}>0.06$, then $p_{t}^{\text {miss }} / \sqrt{s}>0.04$.

(c) The event transverse momentum computed without the hadron calorimeter was required to be larger than $0.02 \times \sqrt{s}$. This cut rejects events with a large measured transverse momentum due to fluctuations in the hadron calorimeter.

Figure $1(\mathrm{~d})$ shows the $a_{t}^{\text {miss }}$ distribution after cuts (1) to (5) for the small acoplanarity region.

\footnotetext{
${ }^{4}$ The acoplanarity angle, $\phi_{\text {acop }}$, is defined as $180^{\circ}$ minus the opening angle between the directions of the momentum components of the two lepton candidates in the $x-y$ plane.

${ }^{5}$ The acollinearity angle, $\phi_{\mathrm{acol}}$, is defined as $180^{\circ}$ minus the three-dimensional angle between the directions of the momentum vectors of the two lepton candidates.
} 
(8a) To reduce the background due to ee $\mu \mu$ events where one electron and one muon escape detection, a kinematic cut was applied when one electron and one muon are identified in the final state, with both leptons having $4<p<15 \mathrm{GeV}$ and $2<p_{t}<10 \mathrm{GeV}$. In this type of ee $\mu \mu$ event, the undetected muon is emitted at a small polar angle while the undetected electron escapes down the beam pipe. The momentum direction of the undetected muon is calculated using total momentum conservation and assuming the existence of an electron with the beam energy emitted along the $z$ axis in the opposite direction to the observed electron. Events with such a "calculated muon track" emitted with a polar angle $\theta$ such that $|\cos \theta|>0.98$ were rejected. This cut affects only the stau search and reduces the estimated background in this channel.

(8b) The background from two-photon processes and $\mathrm{W}^{+} \mathrm{W}^{-}$pair production was further reduced by requiring that the two identified leptons be two electrons or two muons for the selectron and smuon searches, respectively. This selection criterion was not applied in the stau search.

The numbers of events remaining after each cut are listed in Table 1. For comparison, the table also shows the corresponding numbers of simulated events for background processes and the detection efficiencies for three samples of simulated $\tilde{\ell}^{+} \tilde{\ell}^{-}$events. The poor agreement prior to cut (4) between the data and Monte Carlo expectation is due partly to beam related backgrounds and partly to missing simulated events for hadronic two-photon processes in the region of $m_{\gamma \gamma}^{2}<4 \mathrm{GeV}^{2}$ with $Q^{2}<1 \mathrm{GeV}$ and $m_{\gamma \gamma}^{2}<3 \mathrm{GeV}^{2}$ with $Q^{2}>1 \mathrm{GeV}$ and to incomplete modelling of two-photon processes. After cut (4) when the two-photon processes have been effectively reduced, the agreement between data and Monte Carlo is good. Using $10.1 \mathrm{pb}^{-1}$ of data, the expected background from all Standard Model processes considered is estimated to be 3.23 events for the $\tilde{\tau}$ search, 2.73 of which are due to four-fermion processes. Of these 2.73 events $\mathrm{W}^{+} \mathrm{W}^{-}$semileptonic decays are the dominant source. For the same integrated luminosity, the expected total background from Standard Model processes is estimated to be 1.37 events for the $\tilde{\mathbf{e}}$ and $\tilde{\mu}$ searches together, 1.24 of which are due to four-fermion processes.

\section{$4.4 \mathrm{~W}^{+} \mathrm{W}^{-}$Background Reduction}

The number of background events remaining after the selection cuts described in Section 4.3 is composed of a rather large contamination from $\mathrm{W}^{+} \mathrm{W}^{-}$leptonic decays. The momentum distributions of leptons resulting from slepton decays are peaked at different values when compared to those from the $\mathrm{W}^{+} \mathrm{W}^{-}$leptonic decays, especially for small $\Delta m$ values. To reduce the $\mathrm{W}^{+} \mathrm{W}^{-}$background, cuts on the visible energy of the event, $E_{\mathrm{vis}}$, and on the momenta of the more energetic lepton and on the less energetic lepton of the event in the $\left(E_{\mathrm{vis}}, p_{\ell}\right)$ plane (where $p_{\ell}$ is the lepton momentum) can be applied for each lepton. Those cuts reduce the $\mathrm{W}^{+} \mathrm{W}^{-}$background while they reduce by a much smaller amount the efficiencies for selecting slepton pairs. Identical cuts have been applied for the $\tilde{\mathbf{e}}^{+} \tilde{\mathbf{e}}^{-}$and $\tilde{\mu}^{+} \tilde{\mu}^{-}$searches. For the $\tilde{\tau}^{+} \tilde{\boldsymbol{\tau}}^{-}$ search, due to the additional presence of neutrinos in the final state, more stringent cuts in the $\left(E_{\mathrm{vis}}, p_{\ell}\right)$ plane have been applied. The cuts defined to reduce the $\mathrm{W}^{+} \mathrm{W}^{-}$background are summarized in Table 2.

Some typical efficiencies after the $\mathrm{W}^{+} \mathrm{W}^{-}$background reduction cuts for selecting selectron, smuon and stau pair events are summarized in Table 3. The quoted errors are systematic uncertainties due to Monte Carlo statistics. 


\begin{tabular}{|l||r||r||r|r|r||c|c|c|}
\hline & Data & $\begin{array}{c}\text { Total } \\
\text { Backg. }\end{array}$ & $\mathrm{ff}(\gamma)$ & $\mathrm{e}^{+} \mathbf{e}^{-} \mathrm{ff}$ & 4-fermion & $\begin{array}{c}\tilde{\mathbf{e}}^{+} \tilde{\mathbf{e}}^{-} \\
(\%)\end{array}$ & $\begin{array}{c}\tilde{\mu}^{+} \tilde{\mu}^{-} \\
(\%)\end{array}$ & $\begin{array}{c}\tilde{\tau}^{+} \tilde{\tau}^{-} \\
(\%)\end{array}$ \\
\hline$m_{\tilde{\ell}}(\mathrm{GeV})$ & & & & & & 75 & 75 & \multicolumn{1}{c}{75} \\
$\Delta m(\mathrm{GeV})$ & & & & & & \multicolumn{1}{c}{$\begin{array}{c}m_{\tilde{\mathbf{e}}} / 2 \\
m_{\tilde{\mu}} / 2\end{array}$} & $m_{\tilde{\tau}} / 2$ \\
\hline Preselection & 10683 & 6678.46 & 406.11 & 6247.52 & 24.83 & 91.1 & 93.2 & 89.4 \\
cut (1) & 4839 & 3336.36 & 306.95 & 3017.95 & 11.46 & 90.5 & 92.6 & 88.9 \\
cut (2) & 3009 & 2168.89 & 138.83 & 2022.41 & 7.65 & 82.4 & 83.3 & 81.2 \\
cut (3) & 2878 & 2094.02 & 66.49 & 2021.64 & 5.89 & 82.4 & 83.3 & 81.2 \\
cut (4) & 611 & 639.12 & 38.94 & 596.03 & 4.15 & 78.9 & 80.0 & 60.3 \\
cut (5) & 179 & 188.46 & 4.33 & 180.79 & 3.34 & 73.8 & 73.3 & 54.4 \\
cut (6) & 177 & 185.16 & 1.08 & 180.78 & 3.30 & 73.1 & 73.3 & 53.3 \\
cut (7) & 4 & 3.55 & $\mathbf{0 . 1 8}$ & $\mathbf{0 . 6 3}$ & 2.74 & 70.2 & 72.9 & 39.9 \\
cut (8a) & 4 & 3.23 & $\mathbf{0 . 1 8}$ & $\mathbf{0 . 3 2}$ & 2.73 & 70.2 & 72.9 & 39.3 \\
cut (8b) & 2 & 1.37 & $\mathbf{0 . 0 8}$ & $\mathbf{0 . 0 6}$ & 1.24 & 68.8 & 72.8 & 39.3 \\
\hline
\end{tabular}

Table 1: The remaining numbers of events normalized to the integrated luminosity of the data for various Monte Carlo background processes are compared with data (first column) after each cut. Efficiencies for three simulated event samples of $\tilde{\mathrm{e}}^{+} \tilde{\mathbf{e}}^{-}$, $\tilde{\mu}^{+} \tilde{\mu}^{-}$and $\tilde{\tau}^{+} \tilde{\tau}^{-}$are also given (last three columns) for $m_{\tilde{\ell}}=75 \mathrm{GeV}$ and $\Delta m=$ $\left(m_{\tilde{\ell}}-m_{\tilde{\chi}_{1}^{0}}\right)=37.5 \mathrm{GeV}$. The poor agreement at the early stages of the analysis is detailed in the text.

Table 4 gives the total background expectation from all Standard Model processes using an integrated luminosity of $10.1 \mathrm{pb}^{-1}$ for the five $\Delta m$ values considered for the selectron, smuon and stau searches, respectively.

The systematic errors on the number of signal events expected that have been considered are: the statistical error on the determination of efficiency from the $\tilde{\ell}^{ \pm}$Monte Carlo simulation; the systematic error on the integrated luminosity of $0.6 \%$ [23]; the systematic error due to the trigger efficiency was estimated to be negligible because of the lepton transverse momentum requirement; the inefficiency due to forward detectors false vetoes was estimated to be $2.3 \%$; the uncertainty due to the interpolation of the efficiencies was estimated to be $2 \%$; the lepton identification uncertainty was estimated to be $3.5 \%$; the systematic uncertainty due to different angular distributions (possible for selectron pair production via the $t$-channel) of produced

\begin{tabular}{|c||c|c||c|c||c|c||c|c||c||c||}
\hline \multicolumn{1}{|c||}{$\Delta m(\mathrm{GeV})$} & \multicolumn{2}{|c||}{$E_{\mathrm{vis}}^{\min } / \sqrt{s}$} & \multicolumn{2}{|||}{$E_{\mathrm{vis}}^{\max } / \sqrt{s}$} & \multicolumn{2}{|c||}{$p_{2}^{\max } / E_{\text {beam }}$} & \multicolumn{2}{|c||}{$p_{1}^{\min } / E_{\text {beam }}$} & \multicolumn{2}{|c||}{$p_{1}^{\max } / E_{\text {beam }}$} \\
\hline & $\tilde{\mathbf{e}}, \tilde{\mu}$ & $\tilde{\tau}$ & $\tilde{\mathbf{e}}, \tilde{\mu}$ & $\tilde{\tau}$ & $\tilde{\mathbf{e}}, \tilde{\mu}$ & $\tilde{\tau}$ & $\tilde{\mathbf{e}}, \tilde{\mu}$ & $\tilde{\tau}$ & $\tilde{\mathbf{e}}, \tilde{\mu}$ & $\tilde{\tau}$ \\
\hline $3^{a)}$ & - & - & 0.4 & 0.15 & 0.10 & 0.10 & - & - & 0.15 & 0.15 \\
5 & - & - & 0.5 & 0.25 & 0.20 & 0.15 & - & - & 0.25 & 0.25 \\
10 & - & - & 0.6 & 0.25 & 0.30 & 0.20 & - & - & 0.50 & 0.25 \\
$m_{\tilde{\ell}} / 2$ & - & - & - & 0.40 & - & 0.30 & - & 0.05 & - & 0.50 \\
$m_{\tilde{\ell}}$ & - & 0.04 & - & 0.50 & - & 0.35 & - & 0.05 & - & 0.50 \\
\hline
\end{tabular}

Table 2: Definition of the cuts used to reduce the background from $\mathrm{W}^{+} \mathrm{W}^{-}$pairs for five $\Delta m$ values. The cuts are optimized separately for the smuon and selectron searches, and for the stau search. $E_{\text {vis }}$ is the event visible energy, $p_{1}$ is the momentum of the more energetic lepton and $p_{2}$ is the momentum of the less energetic lepton. ${ }^{a)}$ For the selectron case the $\Delta m$ value that was generated was $2.5 \mathrm{GeV}$ instead of $3 \mathrm{GeV}$. 


\begin{tabular}{|c|c||c|c|c|}
\hline \multicolumn{2}{|c||}{} & $\tilde{\mathbf{e}}^{+} \tilde{\mathbf{e}}^{-}$ & $\tilde{\mu}^{+} \tilde{\mu}^{-}$ & $\tilde{\boldsymbol{\tau}}^{+} \tilde{\boldsymbol{\tau}}^{-}$ \\
\hline$m_{\tilde{\ell}}(\mathrm{GeV})$ & $\Delta m(\mathrm{GeV})$ & Efficiency (\%) & Efficiency (\%) & Efficiency (\%) \\
\hline \hline 60 & $3^{a)}$ & $18.5 \pm \mathbf{0 . 9}$ & $27.4 \pm 1.4$ & $\mathbf{0 . 8} \pm \mathbf{0 . 3}$ \\
60 & 5 & $41.8 \pm 1.1$ & $48.1 \pm 1.6$ & $7.7 \pm 0.8$ \\
60 & 10 & $58.6 \pm 1.1$ & $64.7 \pm 1.5$ & $22.1 \pm 1.3$ \\
60 & $m_{\tilde{\ell}^{ \pm}} / 2$ & $62.2 \pm 1.1$ & $71.7 \pm 1.4$ & $32.9 \pm 1.5$ \\
60 & $m_{\tilde{\ell}^{ \pm}}$ & $61.1 \pm 1.1$ & $70.8 \pm 1.4$ & $30.7 \pm 1.5$ \\
\hline 75 & $3^{a}$ & $15.2 \pm \mathbf{0 . 8}$ & $29.1 \pm 1.4$ & $\mathbf{0 . 8} \pm \mathbf{0 . 3}$ \\
75 & 5 & $46.3 \pm 1.1$ & $45.5 \pm 1.6$ & $8.2 \pm \mathbf{0 . 9}$ \\
75 & 10 & $64.8 \pm 1.1$ & $63.0 \pm 1.5$ & $22.5 \pm 1.3$ \\
75 & $m_{\tilde{\ell}^{ \pm}} / 2$ & $68.8 \pm 1.0$ & $72.8 \pm 1.4$ & $37.5 \pm 1.5$ \\
75 & $m_{\tilde{\ell}^{ \pm}}$ & $68.2 \pm 1.0$ & $72.7 \pm 1.4$ & $35.9 \pm 1.5$ \\
\hline
\end{tabular}

Table 3: Detection efficiencies in percent for slepton pair production using samples of simulated events for two different selectron, smuon, and stau masses and five different $\Delta m=\left(m_{\tilde{\ell}^{ \pm}}-m_{\tilde{\chi}_{1}^{0}}\right)$ values. ${ }^{a)}$ For the selectron case the $\Delta m$ value that was generated was $2.5 \mathrm{GeV}$ instead of $3 \mathrm{GeV}$. The errors are due to Monte Carlo statistics only.

\begin{tabular}{|c||c|c|c|c|c|c|c|c|c|c|}
\hline \multicolumn{1}{|c||}{ Slepton flavour } & \multicolumn{10}{|c|}{$\Delta m(\mathrm{GeV})$} \\
\cline { 2 - 11 } & \multicolumn{2}{|c|}{$3^{a)}$} & \multicolumn{2}{c|}{5} & \multicolumn{2}{c|}{10} & $m_{\tilde{\ell}} / 2$ & \multicolumn{3}{|c|}{$m_{\tilde{\ell}}$} \\
\hline$\tilde{\mathbf{e}}^{+} \tilde{\mathbf{e}}^{-}$ & $\mathbf{0 . 0 5}$ & $\mathbf{0}$ & $\mathbf{0 . 1 0}$ & 1 & $\mathbf{0 . 3 4}$ & 1 & $\mathbf{0 . 7 0}$ & 1 & $\mathbf{0 . 7 0}$ & 1 \\
$\tilde{\mu}^{+} \tilde{\mu}^{-}$ & 0.0 & $\mathbf{0}$ & $\mathbf{0 . 0 1}$ & $\mathbf{0}$ & $\mathbf{0 . 1 6}$ & $\mathbf{0}$ & $\mathbf{0 . 6 7}$ & 1 & $\mathbf{0 . 6 7}$ & 1 \\
$\tilde{\boldsymbol{\tau}}^{+} \tilde{\boldsymbol{\tau}}^{-}$ & 0.21 & 1 & 0.44 & 1 & $\mathbf{0 . 4 6}$ & 1 & 1.12 & 1 & 1.45 & 2 \\
\hline
\end{tabular}

Table 4: Total expected background and observed events in data for an integrated luminosity of $10.1 \mathrm{pb}^{-1}$ for the selectron, smuon and stau searches for 5 different $\Delta m=\left(m_{\tilde{\ell}}-\right.$ $\left.m_{\tilde{\chi}_{1}^{0}}\right)$ values after having applied cuts in the $\left(E_{\mathrm{vis}}, p_{\ell}\right)$ plane. ${ }^{a)}$ For the selectron case the smallest $\Delta m$ value that was generated was $2.5 \mathrm{GeV}$.

sleptons and decay products was estimated for six different MSSM parameter sets, representing different neutralino field contents (gaugino/higgsino) and couplings, and calculated to be at the level of $1 \%$. The systematic errors were considered to be independent, and the total systematic error was calculated as a quadratic sum of the individual errors. The total systematic error is incorporated into the limits using the method described in Reference [24].

\section{Results}

Four events have been accepted by the cuts described in Section 4.3 after cut $8 \mathrm{a}$. This is compatible with the expected background from Standard Model processes of 3.23 events. One of the events is removed by the $\mathrm{W}^{+} \mathrm{W}^{-}$background reduction criteria for all the slepton channels and for all mass differences. Therefore three events remain, for at least some values of $\Delta m$, after the $\mathrm{W}^{+} \mathrm{W}^{-}$reduction criteria. One event contains two tracks that satisfy the electron identification criteria, one with a momentum of $19.2 \mathrm{GeV}$ and the other one with a momentum of $7.3 \mathrm{GeV}$; it is classified as a selectron candidate for $\Delta m \geq 5 \mathrm{GeV}$; it is also classified as a stau candidate for $\Delta m>m_{\tilde{\ell}} / 2$. This event however contains also an electromagnetic cluster and two energetic hadronic clusters not associated to the two electron tracks which we would not expect 
in a typical selectron pair-produced event. The second event contains two identified muons with momenta of $50.6 \mathrm{GeV}$ and $22.5 \mathrm{GeV}$, respectively; it is classified as a smuon candidate for $\Delta m \geq m_{\tilde{\ell}} / 2$. The third event contains an electron and a tau lepton candidate with momenta of $7.4 \mathrm{GeV}$ and $11.9 \mathrm{GeV}$, respectively, and is classified as a stau candidate for all $\Delta m$ values. As shown in Table 4, the number of events observed in the data is compatible with the expected background from Standard Model sources after $\mathrm{W}^{+} \mathrm{W}^{-}$rejection cuts. In computing limits, no background subtraction was performed. The three events described above have been taken as slepton candidates in the $\left(m_{\tilde{\ell}}, m_{\tilde{\chi}_{1}^{0}}\right)$ regions where they are kinematically compatible by considering the measured momenta of the observed leptons and the errors on the momenta.

Three approaches are used to present slepton production limits. In the first one, upper limits on production cross-sections as a function of slepton and $\tilde{\chi}_{1}^{0}$ masses were calculated with minimal model assumptions. In the second one, limits on the slepton masses were calculated in the framework of the MSSM, under the assumption of a common scalar mass $m_{0}$ at the GUT scale. We have used the branching ratios for the decay $\tilde{\ell}^{ \pm} \rightarrow \ell^{ \pm} \tilde{\chi}_{1}^{0}$ predicted by the MSSM, and we have conservatively assumed no experimental sensitivity to other decay modes. In the third approach, limits on the slepton masses were calculated in the framework of the MSSM, but without the assumption of a common scalar mass at the GUT scale. In this case we have instead assumed mass degeneracy between the right- and left-handed scalar leptons. Following this assumption the total cross-section, $\sigma_{\text {tot }}$, for producing a scalar lepton can be written $\sigma_{\mathrm{tot}}=\sigma_{\mathrm{RR}}+\sigma_{\mathrm{LL}}+2 \cdot \sigma_{\mathrm{LR}}$ for selectron pair-production and $\sigma_{\mathrm{tot}}=\sigma_{\mathrm{RR}}+\sigma_{\mathrm{LL}}$ for scalar muon pair-production, where $\sigma_{\mathrm{RR}}, \sigma_{\mathrm{LL}}$ and $\sigma_{\mathrm{LR}}$ are respectively the production cross-sections for a pair of right-right, left-left and left-right sleptons. As in the second approach we have used the branching ratios for the decay $\tilde{\ell}^{ \pm} \rightarrow \ell^{ \pm} \tilde{\chi}_{1}^{0}$ predicted by the MSSM, and we have conservatively assumed no experimental sensitivity to other decay modes.

Upper limits on the slepton production cross-sections at the $95 \%$ C.L. for $\tilde{\ell}^{+} \tilde{\ell}^{-}$are shown in Fig. 2. These limits do not depend on the details of SUSY models, except for the assumptions that the decay $\tilde{\ell} \rightarrow \ell \tilde{\chi}_{1}^{0}$ has $100 \%$ branching ratio and that the $\tilde{\chi}_{1}^{0}$ does not decay.

The MSSM limits have been computed combining these data collected at $\sqrt{s}=161 \mathrm{GeV}$, together with data previously collected at $\sqrt{s}=130-136 \mathrm{GeV}$ [4]. In the MSSM the pairproduction cross-section for $\tilde{\mathrm{e}}$ is enhanced by the presence of the $t$-channel diagram, especially for $|\mu| \gg M_{2}$ (gaugino region). Figure 3(a) shows the $95 \%$ C.L. exclusion limits for right-handed selectrons, for three possible values of $\mu$ and for $\tan \beta=1.5$. The chosen values of $\mu$ were: -1000 , $-200 \mathrm{GeV}$ and the minimum value ${ }^{6}$ not excluded by OPAL's chargino and neutralino search [25] for this value of $\tan \beta$. The selectron pair-production cross-section decreases with decreasing values of $|\mu|$, therefore the first case of the highest value, at $\mu=-1000 \mathrm{GeV}$, corresponds to the most favourable case, while considering the minimum possible value of $\mu$ results in the smallest number of expected events and the worst case scenario. For the central value of $\mu=-200 \mathrm{GeV}$ and in the case of a massless $\tilde{\chi}_{1}^{0}$, a right-handed selectron with a mass lighter than $71.8 \mathrm{GeV}$ is excluded at the $95 \%$ C.L. When considering the present OPAL mass limit [25] on $\tilde{\chi}_{1}^{0}$ for these MSSM parameters, a $95 \%$ C.L. lower limit on the selectron mass of $71.5 \mathrm{GeV}$ is derived for $m_{\tilde{\chi}_{1}^{0}}$ $=12.0 \mathrm{GeV}$. Since the cross-section for selectron pair-production also increases for increasing

\footnotetext{
${ }^{6}$ For this value of $\tan \beta$, this is the smallest value of $\mu$ allowed by the chargino and neutralino search [25] at $\sqrt{s}=161 \mathrm{GeV}$, assuming that the minimum $m_{0}$ condition in that paper yields the most conservative exclusion region. For a given point in the $\left(m_{\tilde{\chi}_{1}^{0}}, m_{\tilde{e}}\right)$ plane, this minimum $\mu$ value minimizes the right-handed selectron pair production cross-section. We therefore considered that using this minimum $\mu$ value provides the most conservative exclusion region.
} 
values of $\tan \beta$, the limits that we present are valid for any value of $\tan \beta \geq 1.5$. These limits improve on the results described in Reference [4].

With the luminosity available it is now possible to improve on the LEP1 limits on smuon pair-production in the MSSM framework. The cross-section for smuon pair-production does not depend on the value of $\tan \beta$ or of $\mu$. Therefore the limits shown in figure $3(\mathrm{~b})$ are valid for every value of these MSSM parameters. In the case of a massless $\tilde{\chi}_{1}^{0}$, a right-handed smuon with a mass lighter than $48.7 \mathrm{GeV}$ is excluded at the $95 \%$ C.L. When considering the present OPAL mass limit on $\tilde{\chi}_{1}^{0}$ for these MSSM parameters a $95 \%$ C.L. lower limit on the smuon mass of $51.0 \mathrm{GeV}$ is derived for $m_{\tilde{\chi}_{1}^{0}}=12.0 \mathrm{GeV}$.

Due to the lower detection efficiencies we cannot yet improve on the LEP1 limits on scalar tau pair-production.

Figure 4(a) shows the $95 \%$ C.L. exclusion limits for scalar electron pair-production, using a value of $\mu=-200 \mathrm{GeV}$ and for $\tan \beta=1.5$, assuming degenerate masses for $\tilde{\mathrm{e}}_{\mathrm{R}}$ and $\tilde{\mathrm{e}}_{\mathrm{L}}$. Figure 4 (b) shows the $95 \%$ exclusion limits for scalar muon pair-production, assuming degenerate masses for $\tilde{\mu}_{\mathrm{R}}$ and $\tilde{\mu}_{\mathrm{L}}$.

\section{Conclusions}

We have performed a search for charged scalar leptons using the data collected by the OPAL detector at $\sqrt{s}=161 \mathrm{GeV}$ during the summer of 1996 . The experimental signature for $\tilde{\ell}^{+} \tilde{\ell}^{-}$ (where $\tilde{\ell}=\tilde{\mathrm{e}}, \tilde{\mu}, \tilde{\tau})$ is an acoplanar pair of charged leptons $(\ell=e, \mu, \tau)$ with missing momentum carried away by the neutralinos.

No excess of events with these characteristics has been observed in the data. Limits on the pair-production cross-sections for charged scalar leptons have been computed. Exclusion regions have been delineated in the framework of the MSSM. A 95\% C.L. lower limit on the right-handed selectron mass of $71.5 \mathrm{GeV}$ is obtained for $\tan \beta \geq 1.5,|\mu| \geq 200 \mathrm{GeV}$ and $m_{\tilde{\chi}_{1}^{0}}=12.0 \mathrm{GeV}$. A 95\% C.L. lower limit on the right-handed smuon mass of $51.0 \mathrm{GeV}$ is derived for $m_{\tilde{\chi}_{1}^{0}}=12.0 \mathrm{GeV}$. The limit for smuon pair-production does not depend on the value of $\tan \beta$ or $\mu$, and is therefore valid for any values of these parameters. The mass limits for pair-produced left-handed selectrons and smuons would be higher and therefore the limits presented here are to be considered as conservative.

\section{Acknowledgements}

We particularly wish to thank the SL Division for the efficient operation of the LEP accelerator at the new energy of $\sqrt{s}=161 \mathrm{GeV}$ and for their continuing close cooperation with our experimental group. We thank our colleagues from CEA, DAPNIA/SPP, CE-Saclay for their efforts over the years on the time-of-flight and trigger systems which we continue to use. In addition to the support staff at our own institutions we are pleased to acknowledge the Department of Energy, USA, National Science Foundation, USA, Research Corporation, USA, Particle Physics and Astronomy Research Council, UK, 
Natural Sciences and Engineering Research Council, Canada,

Israel Science Foundation, administered by the Israel Academy of Science and Humanities,

Minerva Gesellschaft,

Japanese Ministry of Education, Science and Culture (the Monbusho) and a grant under the

Monbusho International Science Research Program,

German Israeli Bi-national Science Foundation (GIF),

Bundesministerium für Bildung, Wissenschaft, Forschung und Technologie, Germany,

National Research Council of Canada,

Hungarian Foundation for Scientific Research, OTKA T-016660, and OTKA F-015089.

\section{References}

[1] Y. Gol'fand and E. Likhtam, JETP Lett. 13 (1971) 323;

D. Volkov and V. Akulov, Phys. Lett. B46 (1973) 109;

J. Wess and B. Zumino, Nucl. Phys. B70 (1974) 39.

[2] P. Fayet, Unification of the Fundamental Particle Interactions, Plenum Press (1980) 587.

[3] H. P. Nilles, Phys. Rep. 110 (1984) 1;

H. E. Haber and G. L. Kane, Phys. Rep. 117 (1985) 75.

[4] OPAL Collab., G. Alexander et al., "Searches for Supersymmetric Particles and Anomalous Four-Jet Production at $\sqrt{s}=130-136 \mathrm{GeV}$ at LEP", CERN-PPE/96-096, to be published in Z. Phys.

[5] ALEPH Collab., D. Buskulic et al., Phys. Lett. B373 (1996) 246;

ALEPH Collab., D. Buskulic et al., "Mass Limit for the lightest Neutralino", CERNPPE/96-083, submitted to Z. Phys.;

DELPHI Collab., P. Abreu et al., "Search for Neutralinos, Scalar Leptons and Scalar Quarks in $e^{+} e^{-}$Interactions at $\sqrt{s}=130-136 \mathrm{GeV}$ ", CERN-PPE/96-110, submitted to Phys. Lett. ;

L3 Collab., M. Acciarri et al., Phys. Lett. B377 (1996) 289.

[6] OPAL Collab., K. Ahmet et al., Nucl. Instr. Meth. A305 (1991) 275;

P. P. Allport et al., Nucl. Instr. Meth. A324 (1993) 34;

P. P. Allport et al., Nucl. Instr. Meth. A346 (1994) 476.

[7] B.E. Anderson et al., IEEE Transactions on Nuclear Science 41 (1994) 845.

[8] S. Katsanevas and S. Melachroinos, in "Physics at LEP2", eds. G. Altarelli, T. Sjöstrand and F. Zwirner, CERN 96-01, vol. 2, p. 328 (1996).

[9] R. Bhattacharya, J. Smith and G. Grammer, Phys. Rev. D15 (1977) 3267;

J. Smith, J.A.M. Vermaseren and G. Grammer, Phys. Rev. D15 (1977) 3280.

[10] T. Sjöstrand and M. Bengtsson, Comp. Phys. Comm. 43 (1987) 367;

"PYTHIA 5.6 and JETSET 7.3, Physics and Manual", CERN-TH. 6488/92;

T. Sjöstrand, Comp. Phys. Comm. 82 (1994) 74. 
[11] R. Engel, in "Physics at LEPQ", eds. G. Altarelli, T. Sjöstrand and F. Zwirner, CERN 96-01, vol. 2, p. 216 (1996).

[12] S. Jadach, B.F.L. Ward and Z. Wąs, Comp. Phys. Comm. 79 (1994) 503.

[13] S. Jadach, W. Placzek, and B.F.L. Ward, UTHEP/95-1001 (unpublished).

[14] G. Montagna et al., Nucl. Phys. B452 (1994) 161.

[15] F.A. Berends, R. Pittau and R. Kleiss, Comput. Phys. Commun. 85 (1995) 437.

[16] J. Allison et al., Nucl. Instr. Meth. A317 (1992) 47.

[17] OPAL Collaboration, R. Akers et al., Z. Phys. C61 (1994) 19.

[18] S. Robins, "A Study of Bhabha Scattering at LEP", Ph.D. Thesis (1992), Queen Mary and Westfield College (unpublished).

[19] OPAL Collab., M.Z. Akrawy et al., Phys. Lett. B253 (1991) 511.

[20] OPAL Collab., R. Akers et al., Phys. Lett. B327 (1994) 411.

[21] OPAL Collab., R. Akers et al., Z. Phys. C60 (1993) 199.

[22] OPAL Collab., P.D. Acton et al., Z. Phys. C60 (1993) 19.

[23] OPAL Collab., G. Alexander et al., "Production of Fermion-pair Events in $e^{+} e^{-}$Collisions at 161 GeV Centre-of-mass Energy", CERN-PPE/96-156, to be published in Phys. Lett. B.

[24] R. D. Cousins and V. L. Highland, Nucl. Instr. Meth. A320 (1992) 331.

[25] OPAL Collab., G. Alexander et al., "Search for Chargino and Neutralino Production in $e^{+} e^{-}$Collisions at $\sqrt{s}=161 \mathrm{GeV}$ at LEP", CERN-PPE/96-135, to be published in Phys. Lett. B. 

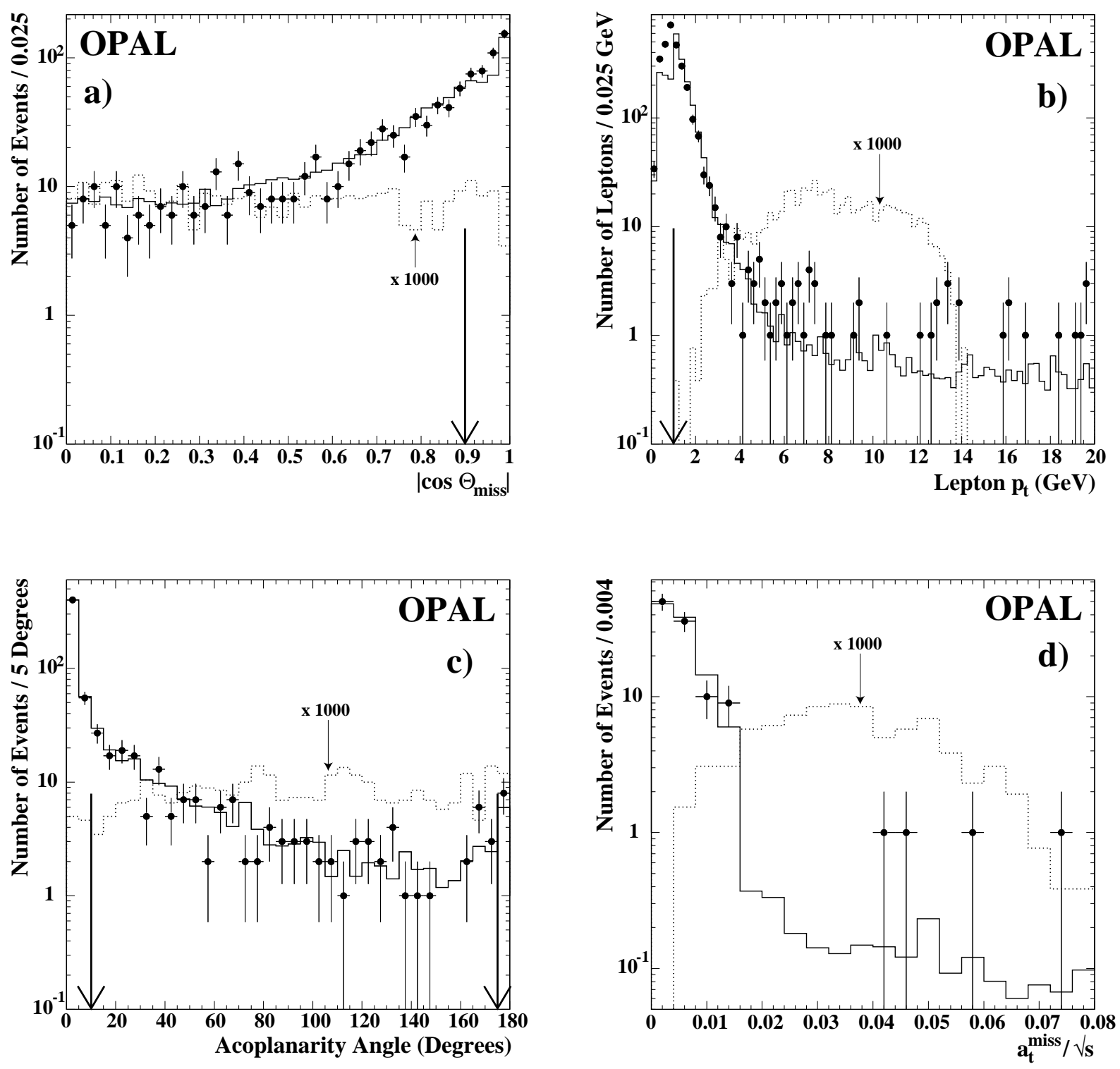

Figure 1: Distributions of (a) the cosine of the polar angle of the missing momentum direction; (b) the transverse momentum, $p_{t}$, of lepton candidates; (c) the acoplanarity angle between the two lepton candidates; and (d) $a_{t}^{\text {miss }}$ of the events for the small acoplanarity region (with no cut on $\left.E_{\text {vis }}\right)$. Data are shown as points and the sum of all Monte Carlo background processes is shown as solid lines. Distributions are shown after certain cuts have been applied as described in the text. The discrepancies observed in some regions of the plots are due to missing simulated events for hadronic two-photon processes in the region of $m_{\gamma \gamma}^{2}<4 \mathrm{GeV}^{2}$ with $Q^{2}<1 \mathrm{GeV}$ and $m_{\gamma \gamma}^{2}<3 \mathrm{GeV}^{2}$ with $Q^{2}>1 \mathrm{GeV}$ and to incomplete modelling of two-photon processes. The dotted histogram shows $\tilde{\mu}^{+} \tilde{\mu}^{-}$Monte Carlo events for $m_{\tilde{\mu}}=75 \mathrm{GeV}$ and $m_{\tilde{\chi}_{1}^{0}}=65 \mathrm{GeV}$. The simulated signal events are normalized using the MSSM predicted cross-section (multiplied by 1000) and the integrated luminosity for this data sample. The arrows indicate the positions of the cuts. 

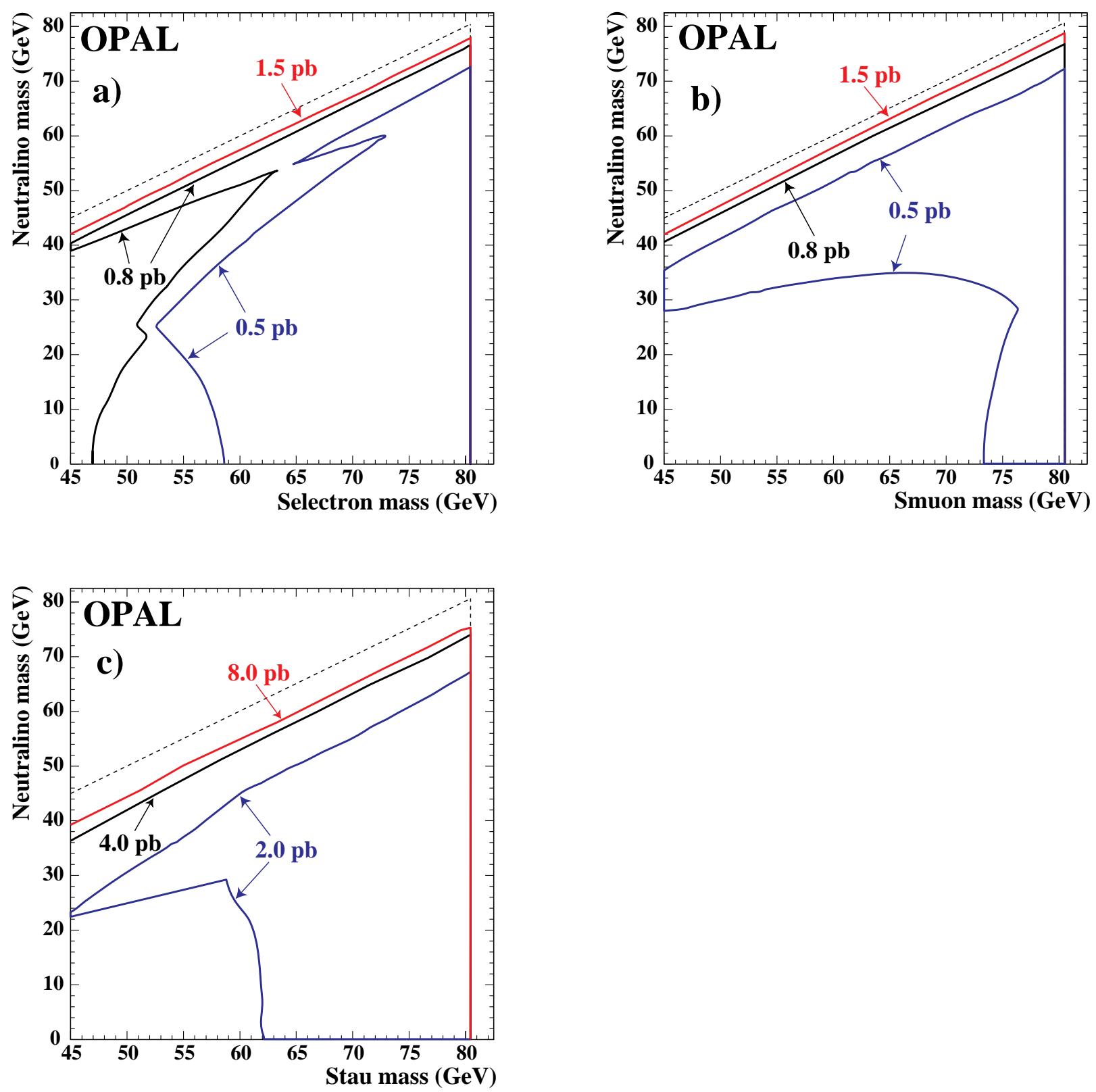

Figure 2: Slepton pair production cross-section upper limits at 95\% C.L. for (a) selectrons, (b) smuons and (c) staus, assuming the decay mode $\tilde{\ell}^{ \pm} \rightarrow \ell^{ \pm} \tilde{\chi}_{1}^{0}$. The kinematic limit at $\sqrt{s}=161 \mathrm{GeV}$ is shown as the dashed line. 

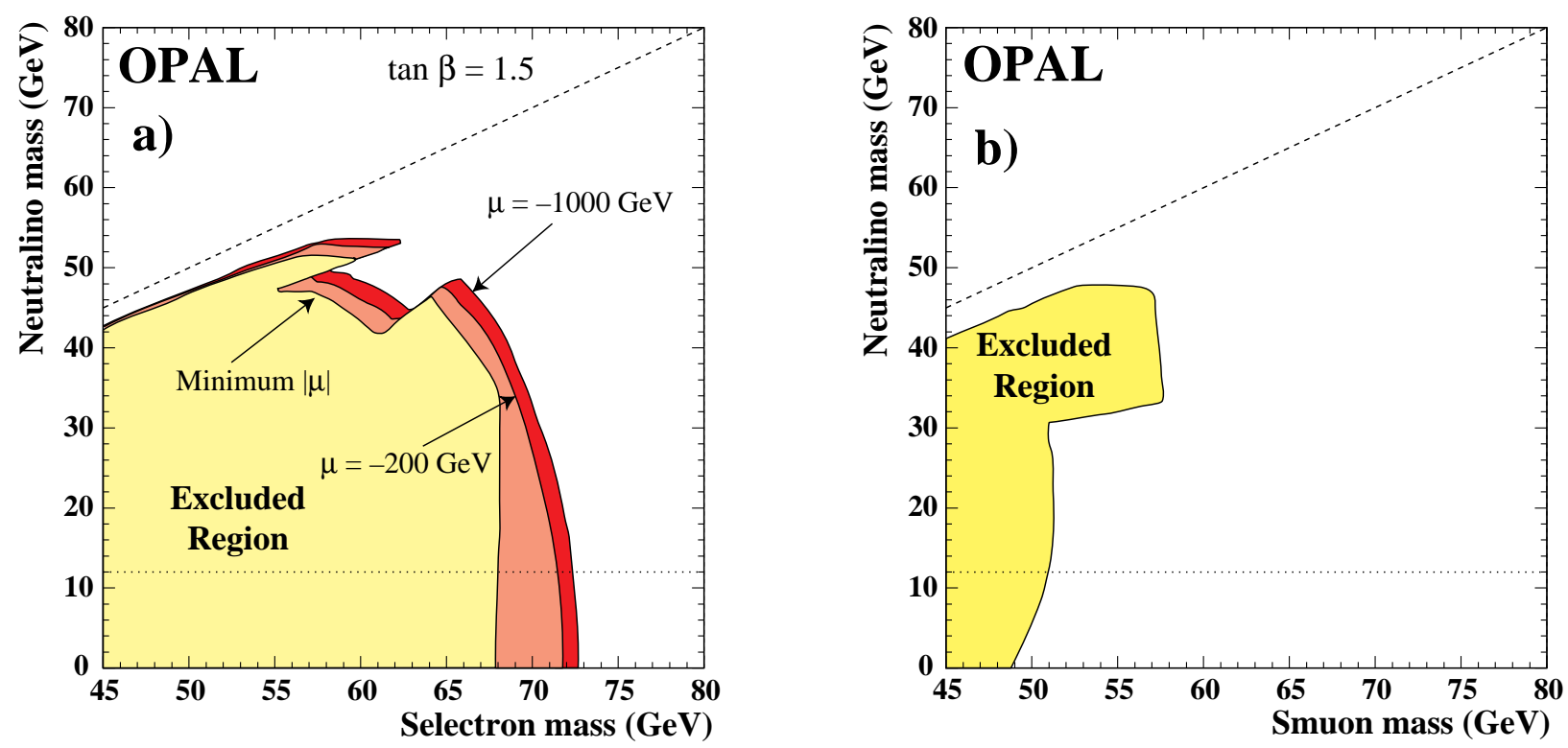

Figure 3: MSSM exclusion region for (a) $\tilde{\mathrm{e}}_{\mathrm{R}}^{+} \tilde{\mathrm{e}}_{\mathrm{R}}^{-}$production in the $\left(m_{\tilde{\mathrm{e}}_{\mathrm{R}}}, m_{\tilde{\chi}_{1}^{0}}\right)$ plane at $95 \%$ C.L. for the three values of $\mu$ described in the text and $\tan \beta=1.5$, using the MSSM predicted production cross-section and branching ratio for $\tilde{\ell}^{ \pm} \rightarrow \ell^{ \pm}+\tilde{\chi}_{1}^{0}$. (b) MSSM exclusion region for $\tilde{\mu}_{\mathrm{R}}^{+} \tilde{\mu}_{\mathrm{R}}^{-}$production in the $\left(m_{\tilde{\mu}_{\mathrm{R}}}, m_{\tilde{\chi}_{1}^{0}}\right)$ plane at $95 \%$ C.L. The kinematic limit is shown as the dashed line. The dotted line shows the current OPAL $\tilde{\chi}_{1}^{0}$ lower mass limit for $\tan \beta=1.5$. These plots include data collected at $\sqrt{s}=161 \mathrm{GeV}$, described in this letter, together with data collected previously at $\sqrt{s}=130-136 \mathrm{GeV}[4]$. 

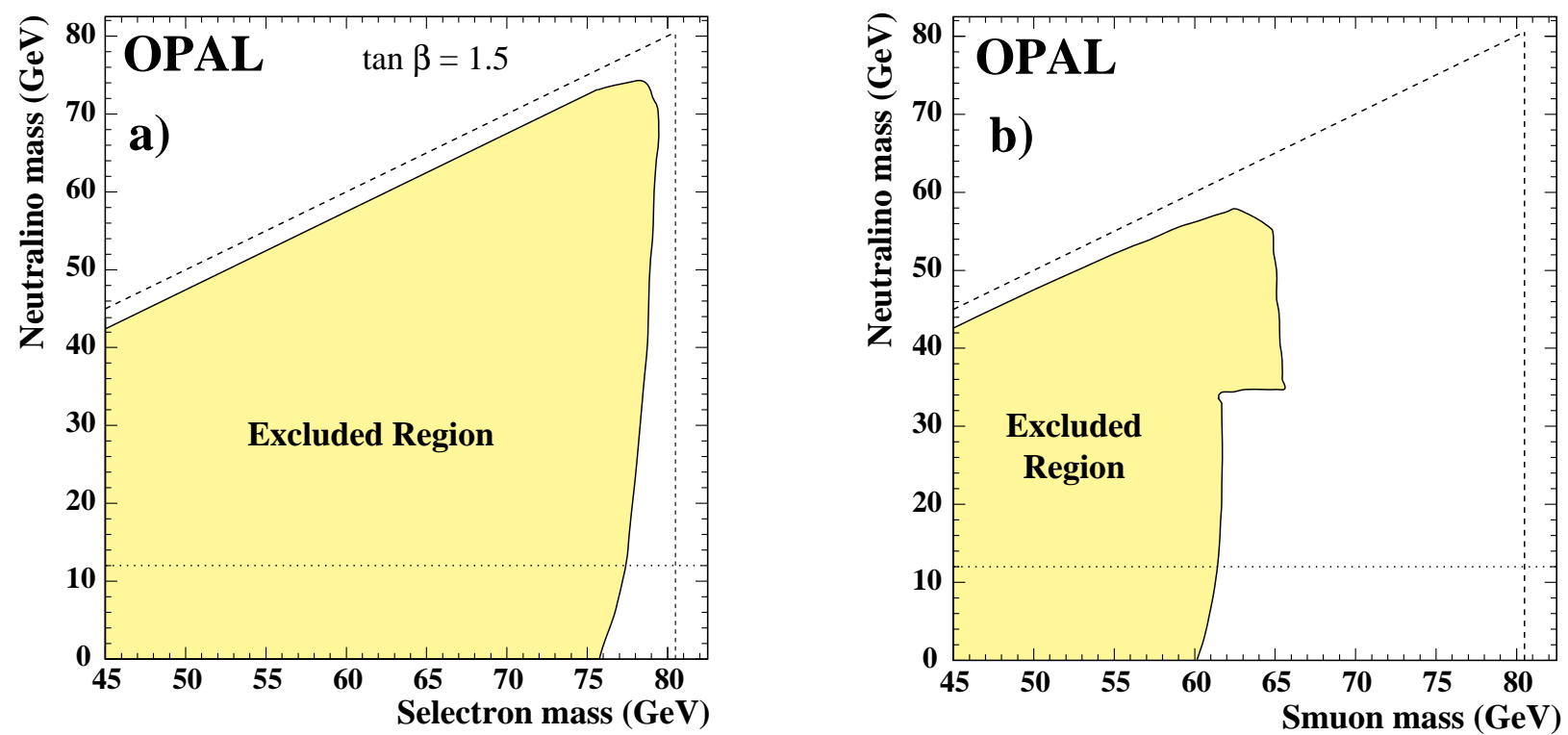

Figure 4: (a) MSSM exclusion region for $\tilde{\mathbf{e}}^{+} \tilde{\mathbf{e}}^{-}$production in the $\left(m_{\tilde{\mathbf{e}}}, m_{\tilde{\chi}_{1}^{0}}\right)$ plane at $95 \%$ C.L. for $\mu=-200 \mathrm{GeV}$ and $\tan \beta=1.5$, assuming mass degeneracy between the right- and left-handed slepton counter-parts, using the MSSM predicted production cross-section and branching ratio for $\tilde{\ell}^{ \pm} \rightarrow \ell^{ \pm}+\tilde{\chi}_{1}^{0}$. (b) MSSM exclusion region for $\tilde{\mu}^{+} \tilde{\mu}^{-}$production in the $\left(m_{\tilde{\mu}}, m_{\tilde{\chi}_{1}^{0}}\right)$ plane at $95 \%$ C.L. assuming mass degeneracy between the right- and left-handed slepton counter-parts. The kinematic limit is shown as the dashed line. The dotted line shows the current $\tilde{\chi}_{1}^{0}$ lower mass limit for $\tan \beta \geq 1.5$. These plots include data collected at $\sqrt{s}=161 \mathrm{GeV}$, described in this letter, together with data collected previously at $\sqrt{s}=130-136 \mathrm{GeV}$ [4]. 